\title{
Glycosylation-site-selective synthesis of $\mathbf{N}$-acetyl-lactosamine repeats in bis-glycosylated human lysozyme
}

\author{
Ralph MELCHER*, Alexandra HILLEBRAND*, Ute BAHR $\dagger$, Bernd SCHRÖDER*, Michael KARAS $\dagger$ and Andrej HASILIK ${ }^{\star 1}$ \\ *Institut für Physiologische Chemie, Philipps-Universität Marburg, D-35033 Marburg, Germany, and †Instrumentelle Analytische Chemie, J. W. Goethe-Universität, \\ D-60590 Frankfurt, Germany
}

\begin{abstract}
We have studied the elongation of oligosaccharides containing $\mathrm{N}$-acetyl-lactosamine repeats using glycosylated human lysozyme mutants as a model. We reported previously that a combination of glycosylation sites at the 49th (site IV) and 68th (site II) amino acid residues of the protein particularly stimulates the synthesis of $N$-acetyl-lactosamine repeats [Melcher, Grosch, Grosse and Hasilik (1998) Glycoconjugate J. 15, 987-993]. In the present study we show that it is the carbohydrate attached to site IV that is selectively affected. It contains more $N$-acetyl-lactosamine
\end{abstract}

repeats when site II is glycosylated in the same molecule. As a corollary of the glycosylation at site II, the synthesis of a third antenna at site IV is increased. The triantennary oligosaccharides at site IV contain more $N$-acetyl-lactosamine repeats than the biantennary ones. Thus placing a carbohydrate at site II stimulates the branching and the elongation of the carbohydrate at the other site.

Key words: mutant, oligosaccharide, polylactosamine.

\section{INTRODUCTION}

Migration of cells from the vasculature into the surrounding environment is an important step in defense reactions and in the spread of metastases (reviewed in [1,2]). During inflammation, endothelium in the affected tissue becomes activated and interacts with cells bearing cognate structures, such as sialyl Lewis ${ }^{\mathrm{x}}$ substances. These substances bind to selectins, which are exposed on the surface of endothelium and platelets, in a regulated manner. They are found at the non-reducing termini of various glycoconjugates with extensions made of $N$-acetyl-lactosamine repeats. It has been shown in several systems that the synthesis of the repeats depends on the expression of distinct enzymes, including the elongating $\beta-1,3-N$-acetylglucosaminyltransferase [3-5] and the branching $\beta-1,6-N$-acetylglucosaminyltransferases [6,7]. Sialyltransferases, meanwhile, act as terminators of elongation [8,9]. $N$-Acetylglucosaminyltransferase $\mathrm{V}$ (responsible for the synthesis of the $\beta$-1,6-linked branch in complex oligosaccharides) is considered to be an important regulator of the $\mathrm{N}$-acetyl-lactosamine repeat synthesis, since branched oligosaccharides are better substrates for elongation than linear ones $[6,7]$. The branching of complex oligosaccharides and the elongation of the repeats are increased in human neoplastic cells [10]. These modifications support the synthesis of sialyl Lewis ${ }^{\mathrm{x}}$ substances and increase the metastatic potential of cells.

Previously we have described glycosylation of lysozyme mutants at sites referred to as I $\left(\mathrm{Asn}^{22}\right)$, II $\left(\mathrm{Asn}^{68}\right)$ and IV $\left(\mathrm{Asn}^{49}\right)$ $[9,11]$. We have shown that the processing of the carbohydrates at these sites is similar. Lysozyme glycosylated at site I has been found to contain predominantly biantennary oligosaccharides bearing zero to four $\mathrm{N}$-acetyl-lactosamine repeats (in order of decreasing frequency). The size of the oligosaccharides appears to be significantly increased in a mutant bearing carbohydrate simultaneously at sites II and IV, but not in a mutant glycosylated at sites I and IV. In the present study we show that the characteristic modification in molecules glycosylated at sites II and IV particularly affects site IV. Using MS analysis of glycopeptides that were prepared from molecules glycosylated at either site alone or at both sites simultaneously, we conclude that glycosylation at site II strongly affects the processing of the oligosaccharides at site IV. These oligosaccharides become enriched in elongated triantennary oligosaccharides.

\section{MATERIALS AND METHODS}

\section{Materials and cells}

Human milk lysozyme and rabbit anti-(human lysozyme) serum have been prepared and characterized previously [9,12]. Radioactive methionine/cysteine mixture ( $\operatorname{Tran}^{35} \mathrm{~S}$-label) was purchased from ICN Biomedicals (Eschwege, Germany). Endo- $\beta$ - $N$ acetylglucosaminidase F2 from Flavobacterium meningosepticum and endo- $\beta$-galactosidase from Bacteroides fragilis were obtained from Boehringer-Mannheim (Mannheim, Germany). Chinese hamster ovary (CHO) cells expressing glycosylated human lysozyme II/IVT have been described previously [11]. The glycosylation sites that are referred to by the symbols II and IVT have been introduced with mutations G68N/P71S and D49N/S51T, respectively. These cells were prepared by stably transfecting $\mathrm{CHO}$ cells (CCL61) that were obtained from the American Type Culture Collection (Manassas, VA, U.S.A.).

\section{Cell culture}

Cells were grown to confluency in $\alpha$-minimum essential medium (Life Technologies, Eggenstein, Germany), supplemented with $10 \%$ (v/v) fetal bovine serum (Sigma, Deisenhofen, Germany), 100 units $/ \mathrm{ml}$ penicillin and $100 \mu \mathrm{g} / \mathrm{ml}$ streptomycin, under air $/ \mathrm{CO}_{2}(19: 1)$ at $37^{\circ} \mathrm{C}$.

\footnotetext{
Abbreviations used: $\mathrm{CHO}$, Chinese hamster ovary; MALDI-TOF MS, matrix-assisted laser-desorption ionization-time-of-flight MS.

1 To whom correspondence should be addressed (e-mail hasilik@mailer.uni-marburg.de).
} 


\section{Metabolic labelling, immunoprecipitation and characterization of the labelled lysozyme}

Cells grown to early confluency in $3.4 \mathrm{~cm}$ diameter dishes were incubated in medium free of methionine and cysteine that was supplemented with $4 \mathrm{MBq}$ of the Tran ${ }^{35} \mathrm{~S}$-label for $16 \mathrm{~h}$. Labelled lysozyme was isolated from the medium by immunoprecipitation [9], separated by SDS/PAGE [13] and visualized by PhosphorImager analysis (Molecular Dynamics, Krefeld, Germany). The quantification of the radioactivity associated with lysozyme was evaluated with software provided by the supplier. To characterize the carbohydrate moiety of lysozyme, aliquots of the immunoprecipitates were treated with endo- $N$-acetylglucosaminidase F2 and endo- $\beta$-galactosidase [9].

\section{Purification and characterization of lysozyme II/IVT}

Recombinant lysozyme II/IVT was produced in cultures of transfected CHO cells and purified from the conditioned medium using immune-affinity and hydroxylapatite chromatography. A previously described procedure [11] was scaled up to obtain $100-200 \mu \mathrm{g}$ of protein. Briefly, $200 \mu \mathrm{g}$ of affinity purified rabbit anti-(human lysozyme) antibody was coupled to $\mathrm{CNBr}$-activated Sepharose 4B (Amersham Pharmacia Biotech, Braunschweig, Germany). Medium containing lysozyme was applied to the immunosorbent in a column and the enzyme was eluted with $0.1 \mathrm{M}$ glycine $/ \mathrm{HCl}(\mathrm{pH} 2.65) / 2 \mathrm{M} \mathrm{MgCl}$. After concentrating and dialysing the eluate against $10 \mathrm{mM}$ sodium phosphate buffer (pH 6.8), it was applied to a hydroxyapatite column (CHT2-I from Bio-Rad Laboratories $\mathrm{GmbH}$, München, Germany) and this column was eluted with a linear gradient up to $0.5 \mathrm{M}$ sodium phosphate buffer ( $\mathrm{pH}$ 6.8). Mixtures of glycosylated forms of lysozyme were separated by SDS/PAGE and visualized by staining with silver nitrate [14].

\section{Tryptic digestion, isolation and identification of glycopeptides}

Fractions from the hydroxylapatite column, containing monoand bis-glycosylated lysozyme, were pooled and vacuum-dried. The residues were dissolved in $0.3 \mathrm{ml}$ of $5 \mathrm{M}$ guanidinium chloride. The solution was mixed with 0.1 vol. of $1 \mathrm{M}$ dithiothreitol for $30 \mathrm{~min}$ at $37^{\circ} \mathrm{C}$, followed by $0.15 \mathrm{vol}$. of $2 \mathrm{M}$ iodoacetic acid (that was neutralized with $\mathrm{NaOH}$ ) for 30 min at $37{ }^{\circ} \mathrm{C}$. The solution was then dialysed against $2 \mathrm{M}$ urea at $4{ }^{\circ} \mathrm{C}$. The resulting solution was subsquently diluted with 1 vol. of $\mathrm{H}_{2} \mathrm{O}$ and buffered by adding $0.25 \mathrm{vol}$. of $0.5 \mathrm{M}$ Tris $/ \mathrm{HCl}(\mathrm{pH}$ 8.5). The carboxymethylated protein was digested with $4 \mu \mathrm{g}$ of sequencing-grade trypsin (Boehringer Mannheim) at $37^{\circ} \mathrm{C}$ for $16 \mathrm{~h}$. The digested mixture was then vacuum-dried and separated by HPLC using a $2 \mathrm{~mm} \times 150 \mathrm{~mm} \mathrm{C}_{18}$ reverse-phase column (300 $\AA$ pore size, $5 \mu \mathrm{m}$ bead diameter; Delta-Pak from J. T. Baker, Phillipsburg, NJ, U.S.A.). A linear gradient up to $70 \%$ $(\mathrm{v} / \mathrm{v})$ acetonitrile over $90 \mathrm{~min}$ in $0.5 \%$ trifluoroacetic acid $(\mathrm{pH}$ 2.0) was used in a Waters 625LC system with the PDA Waters 996UV detector (Millipore Waters, Eschborn, Germany). Rechromatography was performed at $\mathrm{pH} 6$ in $0.1 \%$ (w/v) ammonium acetate with a linear gradient up to $70 \%(\mathrm{v} / \mathrm{v})$ acetonitrile in this buffer over $90 \mathrm{~min}$. For comparative purposes, human milk lysozyme [12] was digested and the peptide fragments were separated as above. The glycopeptides were identified by amino acid sequencing that was performed in a Model 477A amino acid sequencer (Applied Biosystems, Weiterstadt, Germany).

\section{Mass analyses}

Mass analyses of the glycopeptides were carried using a matrixassisted laser-desorption ionization-time-of-flight (MALDI-
TOF) $[15,16]$ mass spectrometer (Voyager RP; Perseptive Biosystems, Framingham, MA, U.S.A.). For mass measurements samples were dissolved in a mixture of acetonitrile $/ 0.1 \%$ aqueous trifluoroacetic acid $(1: 1, \mathrm{v} / \mathrm{v})$ at a concentration of approx. $10^{-6}$ M. Samples $(1 \mu \mathrm{l})$ were mixed with $1 \mu \mathrm{l}$ of matrix [2,5-dihydroxybenzoic acid/2-hydroxy-5,5-methoxybenzoic acid; $9: 1$, by vol. ( $20 \mathrm{~g} / 1$ of each acid was dissolved in the sample solvent)] directly on the metal sample support and dried in a stream of cold air. Positive ions were analysed in the linear mode. For each spectrum $30-50$ single mass scans were accumulated. Mass calibration was performed externally with insulin as the calibrant. Peak intensities were taken as arbitary units for the maximum peak heights evaluated by the instrumental software (Grams/386).

\section{RESULTS}

\section{Isolation of glycopeptides from mono- and bis-glycosylated Iysozyme}

Mutant lysozyme II/IVT with glycosylation sites at $\mathrm{Asn}^{49}$ (site IV) and $\mathrm{Asn}^{68}$ (site II) was expressed in stably transfected CHO cells. Glycosylation site IV has been engineered in analogy to the single glycosylation signal in lactoglobulin, a similar protein. An additional mutation, S51T, has been introduced to obtain better utilization of the glycosylation signal [11]. The recombinant glycoprotein was purified from conditioned medium using immune-affinity and hydroxyapatite column chromatography. Aliquots of the eluted fractions were analysed by SDS/PAGE. Figure 1 shows that molecules with the highest apparent size and contents of carbohydrate were eluted from the hydroxyapatite column first. The bands representing lysozyme with different contents of carbohydrate were interpreted according to the previously published characterization [11]. Fraction numbers 16-22 and 24-26, which contained the bulk of the bis- and monoglycosylated molecules, respectively, were pooled and the materials were subjected to tryptic digestion. The apparent crosscontamination of mono- and bis-glycosylated lysozyme in the hydroxyapatite column fractions was considered tolerable for the characterization of the pooled fractions. Owing to a high efficiency of glycosylation of lysozyme II/IVT, the non-glycosylated form of the mutant could not be prepared with a sufficient yield. Therefore, as a control, wild-type human lysozyme was used.

The digested peptide mixtures were separated by reverse-phase HPLC. Novel peaks were expected in the elution profiles of the glycosylated samples when compared to the control. Although, (1) the control differed from the mutant at four amino acid residues that were likely to affect the elution of the fragments, (2) the glycosylation occurred in the neighbourhood of arginine and lysine residues such that it interfered with the tryptic digestion (see below) and (3) the glycosylation was incomplete, a straightforward comparison was possible of the HPLC profiles shown in Figure 2. The glycosylated materials yielded two additional broad peak areas that were not matched in the control and, therefore, tentatively referred to as glycopeptide pools II and IV. The appearance of a broad peak was particularly striking in the tryptic digest of bis-glycosylated lysozyme, in fractions that eluted at the earlier time (pool IV). Material eluting at the later time absorbed light at $295 \mathrm{~nm}$ and was referred to as glycopeptide II, since from the primary sequence of lysozyme the fragment bearing glycosylation site II was predicted to contain tryptophan (residue 64). The origin and the nomenclature of the glycopeptides from mono- and bis-glycosylated lysozyme fractions were established after determining the amino acid sequence (see below) and are explained in Figure 3. The glycopeptides were re- 


\section{Fraction (No.)}

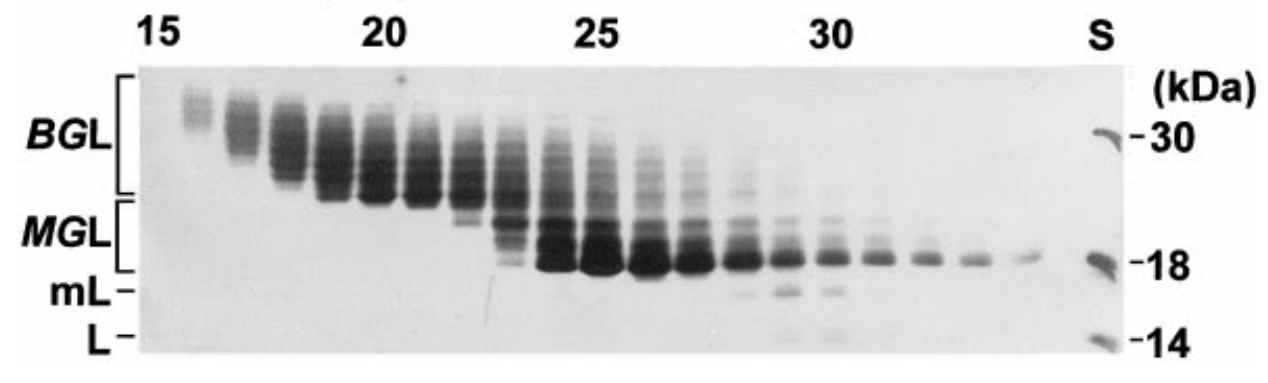

\section{Figure 1 Separation of mono- and bis-glycosylated lysozyme}

Lysozyme mutant II/IVT was purified by immune-affinity and hydroxyapatite chromatography. Aliquots of the fractions of the enzyme that were eluted from the hydroxyapatite column were analysed by SDS/PAGE. Protein was stained with silver nitrate. Carbonic anhydrase, trypsin inhibitor and lysozyme (30, 18 and $14 \mathrm{kDa}$, respectively) were used as standards (S). The positions of lysozyme forms bearing no (L), one mannose-rich (mL), one complex (MGL) or two complex oligosaccharides (BGL) are indicated.

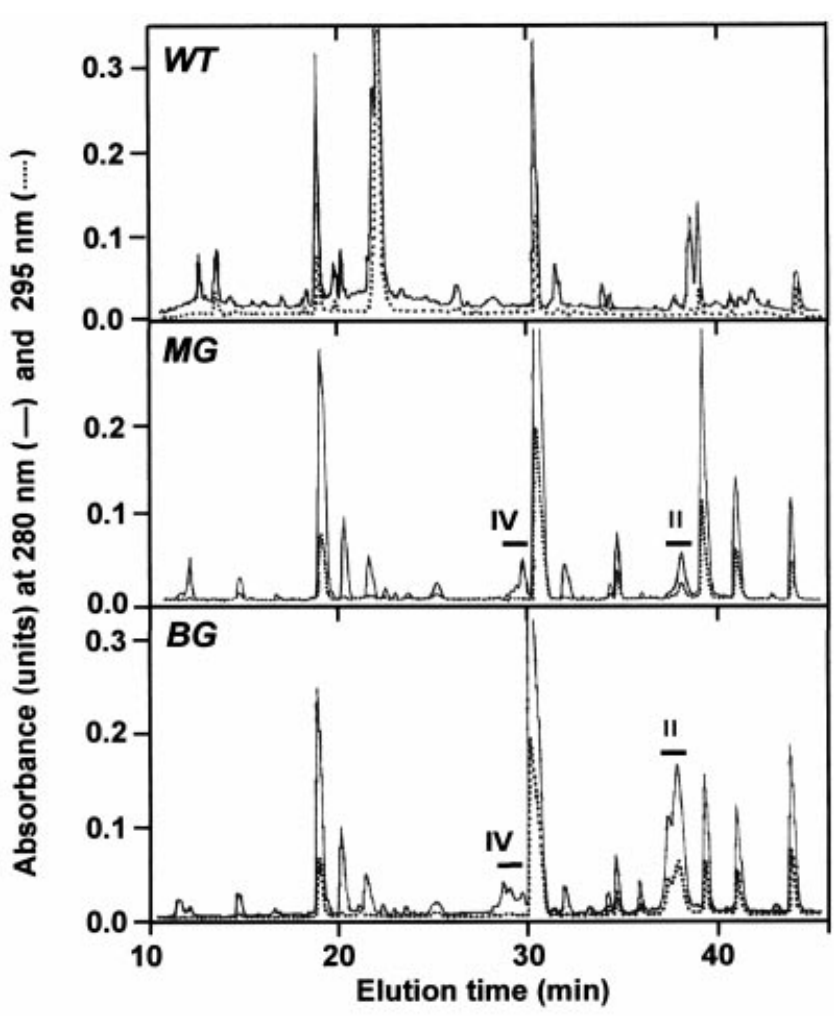

Figure 2 HPLC of tryptic peptides from bis-glycosylated lysozyme

Tryptic peptides were prepared by digesting human milk lysozyme (WT) and bis- (BG) and mono-glycosylated (MG) lysozyme, that corresponded to hydroxylapatite column fractions 16-22 and 24-26, respectively (see Figure 1). The appropriately labelled panels show the separation of the peptides in the three preparations by reverse-phase HPLC. The positions of glycopeptide pools II and IV are indicated. The absorbance at $280 \mathrm{~nm}$ and $295 \mathrm{~nm}$ is represented by the continuous and dotted lines, respectively.

chromatographed at a higher $\mathrm{pH}$. This resulted in the separation of a few minor contaminants (results not shown). In all these cases the major materials were eluted as rather broad peaks. Glycopeptides comprising the glycosylation site II from either mono- or bis-glycosylated lysozyme yielded two broad peaks in tandem, IIA and IIB. They appeared in similar amounts and were pooled and analysed separately. They were assumed to differ from each other in the length of their peptide moieties (see below). Even when subjected to re-chromatography the glycopeptides eluted as rather broad peaks and the heterogeneity was assumed to be due to carbohydrates. The heterogeneity was prominent in the elution profile of glycopeptide IVBG from bisglycosylated lysozyme. In this respect glycopeptide IVBG was clearly distinct from all others including the glycopeptide IVMG that originated from mono-glycosylated lysozyme (Figure 2).

By definition, digestion of bis-glycosylated lysozyme yielded two glycopeptides, IIBG and IVTBG, in equimolar amounts. The ratio of their peak areas was 3.3:1 (Figure 2, lower panel). The apparently higher absorbance of the material in peak II fractions was explained by its larger molecular size. From the ratio of $1.4: 1$ of peak areas of the two glycopeptides in the digest of mono-glycosylated lysozyme (Figure 2, middle panel) it could be concluded that in this mixture molecules glycosylated solely at site II were about half as frequent as those glycosylated solely at site IV. This observation indicated that the two glycosylation sites were utilized independently of each other, since, as reported before [11], in mono-glycosylated lysozyme mutants II and IVT the glycosylation of the former was less frequent.

\section{Identification of the purified glycopeptides}

An aliquot of the re-chromatographed pool IVBG was subjected to automated amino acid sequencing. The sequence obtained in 22 cycles was: ATNYNAG-blank-RTTDYGIFQINSR-blank. It was concluded that this peptide contained glycosylation site IV and that the lack of the signal in the eighth cycle was due to glycosylation of asparagine $\left(\mathrm{Asn}^{49}\right)$ at this position. Thus the glycosylated peptide containing glycosylation site IV comprised 21 amino acid residues (residues 42-62 in lysozyme) and the result of the sequencing confirmed the presence of both mutations (D49N and S51T [11]). It was concluded that the tryptic fragment from pool IVBG was generated by cleavages after $\mathrm{Arg}^{41}$ and $\mathrm{Arg}^{62}$. It was assumed that the lack of cleavage at $\mathrm{Arg}^{50}$ was due to glycosylation at the adjacent mutated site IV (D49N).

A portion of pool IIBGB was subjected to ten cycles of amino acid sequencing with the following result: YWCND-blankKTSG. This sequence corresponded to residues 63-72 of human lysozyme, containing the mutations G68N and P71S and, thus, 


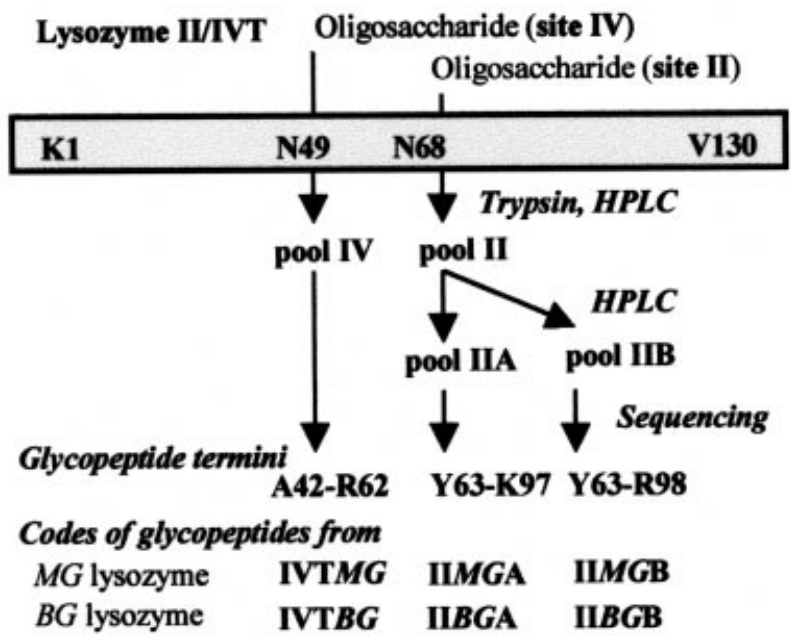

Figure 3 Generation and nomenclature of glycopeptides from glycosylated lysozyme

Glycosylated lysozyme was isolated by immune-affinity chromatography. Mono- and bis-glycosylated lysozyme fractions were separated by hydroxyapatite column chromatography. The glycopeptide codes refer to the origin of two alternative preparations: glycopeptides with codes IVTMG, IIMGA, IIMGB were prepared from a tryptic digest of mono-glycosylated (MG) Iysozyme. Those with codes IVTBG, IIBGA and IIBGB were prepared from bis-glycosylated (BG) lysozyme.

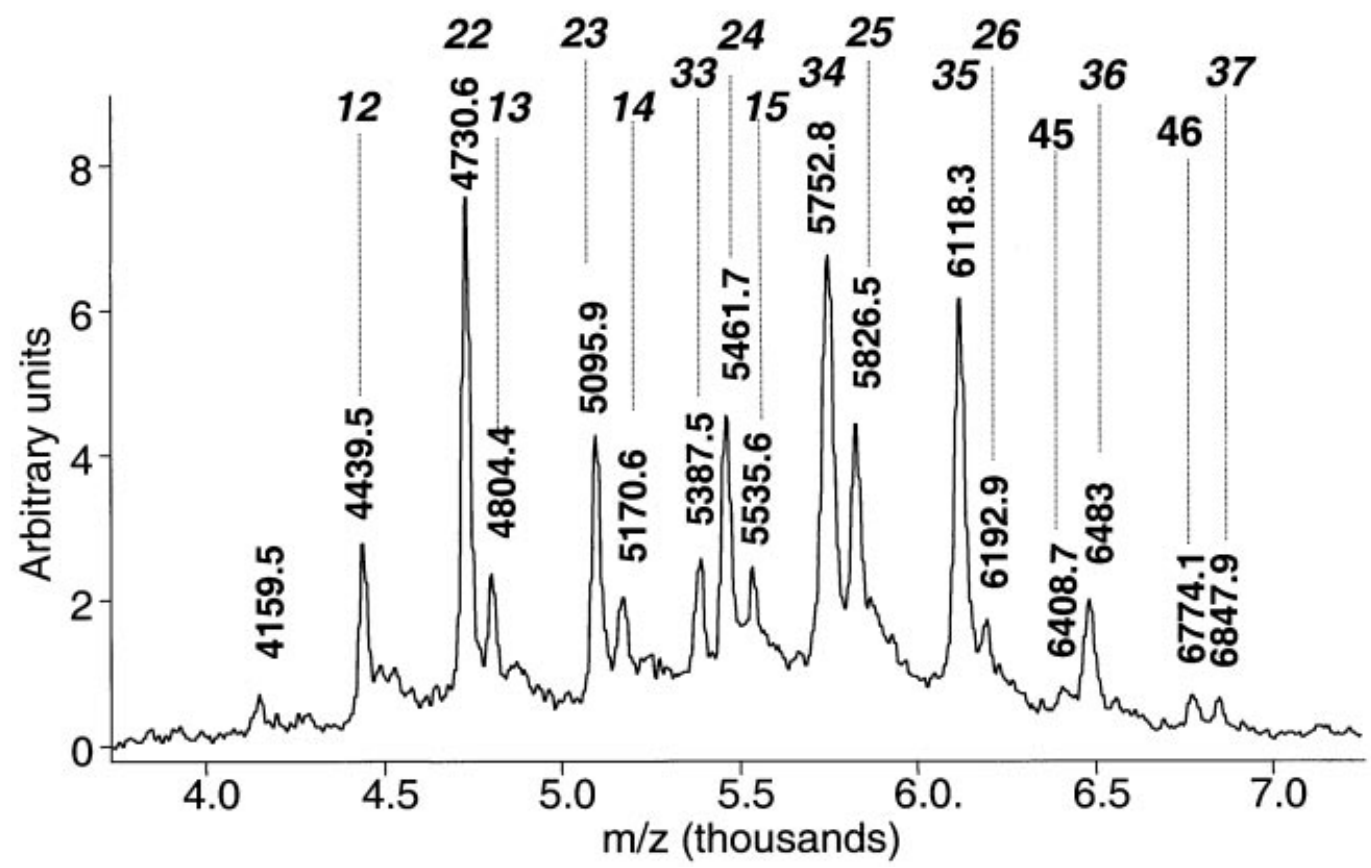

Figure 4 MALDI-TOF mass analysis of tryptic glycopeptide IVBG from glycosylation site IV of bis-glycosylated lysozyme II/IVT molecules

Mass peaks corresponding to glycopeptides bearing mono-, bis- and tris-sialo-oligosaccharides are labelled with numbers that refer to the total content of $\mathrm{N}$-acetylneuraminic acid (i.e. sialic acid) (the first digit) and $\mathrm{N}$-acetyl-lactosamine (the second digit) units. Oligosaccharide structure paradigms are shown in Figure 5.

to glycosylation site II. The sequence was not interrupted after Lys $^{69}$ probably due to glycosylation at the 68 th residue. Mass spectra of glycopeptides IIBGA and IIBGB were similar to each other (see below). The mass values of peaks in the former mass spectrum were $156 \mathrm{Da}$ smaller than in the latter mass spectrum. This difference probably resulted from an alternative cleavage by trypsin at the C-terminal side of the glycopeptide. An aliquot of the peptide IIBGB was subjected to amino acid sequencing to examine this possibility. However, after 32 cycles that confirmed the expected sequence of residues 63-94 the signal ceased to be interpretable. No further attempts to elucidate the mass difference were made. Most probably, the glycopeptides from pool II were generated by tryptic cleavage after $\mathrm{Arg}^{62}$ and either $\mathrm{Lys}^{97}$ or $\mathrm{Arg}^{98}$, and the two alternative fragments were separated during the re-chromatography yielding tandem peaks, IIA and IIB. These optional cleavages are illustrated in Figure 3. 


\section{4}

Biantennary structures
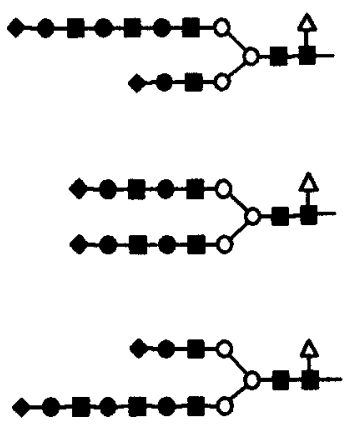

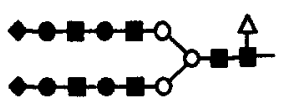

24 and 34

Triantennary structures

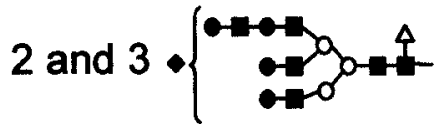
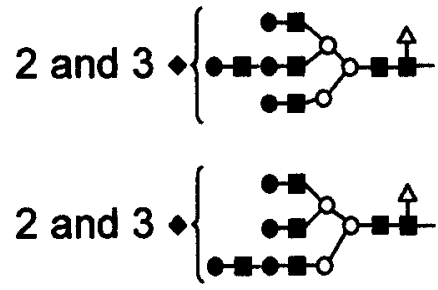

Figure 5 Bi- and tri-antennary isomers of oligosaccharide structures 24 and 34 of glycopeptides from pool IVTBG

Glycopeptide masses 5461.7 Da and 5752.8 Da were examined. The calculated masses of the tryptic peptide $(2374.6 \mathrm{Da})$ and of the fucosylated core carbohydrate mannose $\mathrm{S}_{3}-\mathrm{fucose}_{1}-\mathrm{N}$ acetylglucosamine $_{2}(1039.1 \mathrm{Da})$ moieties were subtracted from the examined values. The residual mass values of 2044.7 Da and 2335.8 Da represented the outer chains of the antennae. These values were compatible with four $\mathrm{N}$-acetyl-lactosamine units (365.4 Da each) and two or three sialic acid residues (291.3 Da each), respectively. Bi- and tri-antennary structures are shown using the following symbols for sialic acid $(\bullet)$, galactose $(\mathbf{O}), N$-acetylglucosamine $(\boldsymbol{\square})$, mannose $(\bigcirc)$ and fucose $(\triangle)$ residues. Tetra-antennary isomers are not shown.
Mass analysis of glycopeptides comprising glycosylation sites II and IVT

MALDI-TOF mass analysis of IVTBG, that contained the glycosylation site IVT and was isolated from bis-glycosylated lysozyme, yielded a complex set of mass signals that are shown in Figure 4. The main peak had a protonated mass of 4730.6. This agreed well with the mass of the mutant sequence $\mathrm{Ala}^{42}$ through to $\mathrm{Arg}^{62}$ (2377.5 Da), combined with that of a biantennary oligosaccharide with two $N$-acetylneuraminic acid, two galactose, three mannose, four $N$-acetylglucosamine and one fucose residues (these residues comprise $2352.2 \mathrm{Da}$ after subtracting the mass of the condensation water), yielding a sum of $4729.7 \mathrm{Da}$.

The peaks shown in Figure 4 were considered to represent glycopeptides with mono- and bis-sialylated biantennary, bisand tris-sialylated triantennary and tetrakis-sialylated tetraantennary oligosaccharides. The peaks were grouped in series that were labelled with two italic numerals. The first numeral was used to indicate the content of sialic acid residues. Within a series the mass values showed an increment that represented the mass of an $\mathrm{N}$-acetyl-lactosamine residue. The total number of $\mathrm{N}$ acetyl-lactosamine units was represented by the second italic numeral. The mass analysis does not distinguish between biantennary oligosaccharides with $n$ lactosamine repeats and triantennary ones with $n-1$ repeats if the two contained less than three sialic acid residues. Correspondingly, the italic code refers to the contents of the two building blocks and not to the number of the antennae. As an example, in Figure 5 biantennary and triantennary isomeric structures are shown that represent oligosaccharide codes 24 and 34 with four $N$-acetyl-lactosamine units and two or three sialic acid residues, respectively.

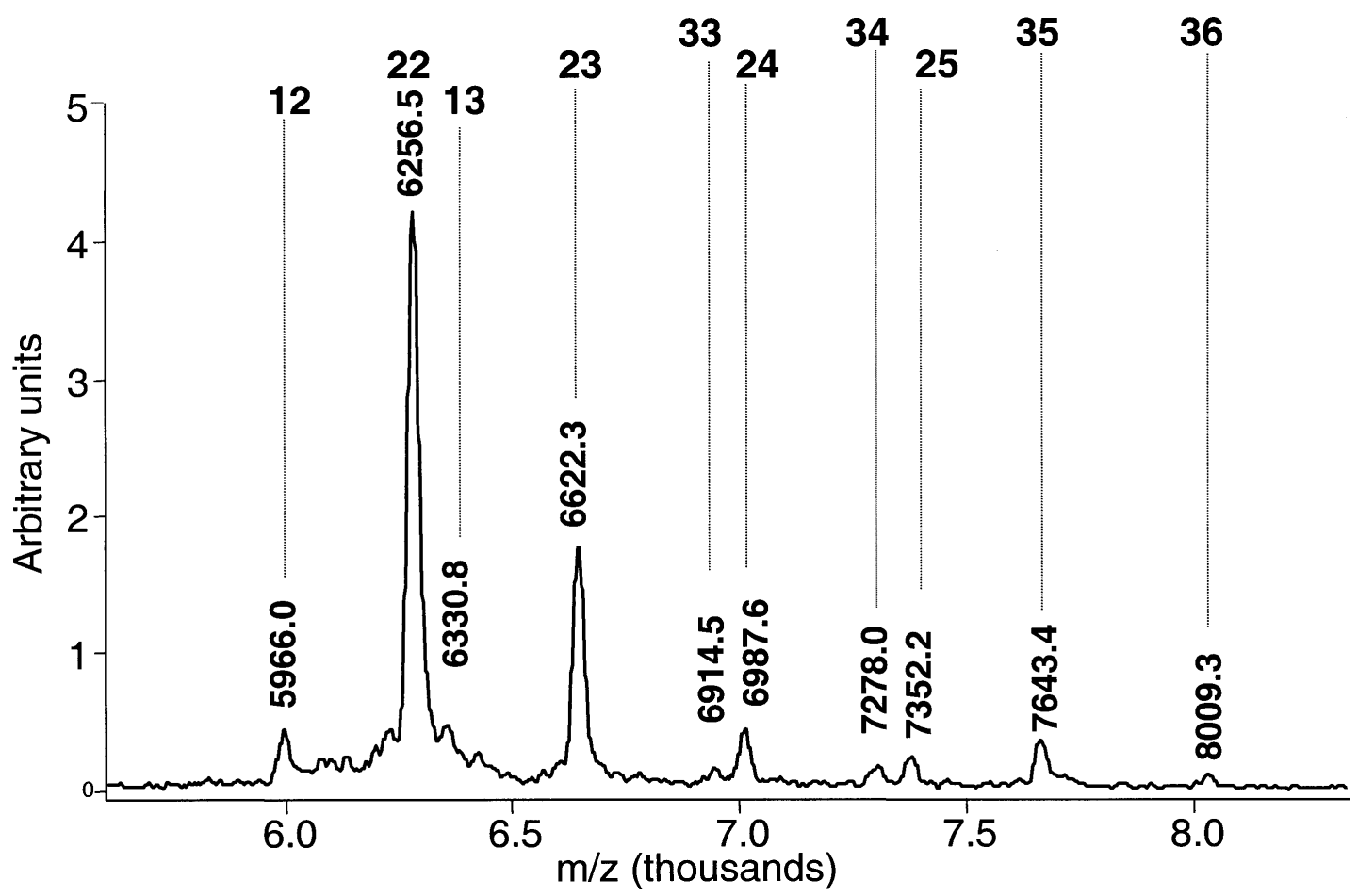

Figure 6 MALDI-TOF mass analysis of tryptic glycopeptide IIBGB from glycosylation site II of bis-glycosylated lysozyme II/IVT molecules

Mass peaks corresponding to glycopeptides with different numbers of $\mathrm{N}$-acetylneuraminic acid and of $\mathrm{N}$-acetyl-lactosamine units are numbered as explained in the legends of Figure 4 and Table 1. 
Table 1 MALDI-TOF mass analysis of glycopeptides from mono- and bisglycosylated lysozyme

In mass spectra of the listed glycopeptides, arbitary units were evaluated that corresponded to calculated masses within $1 \mathrm{Da}$ and represented at least $1 \%$ of the total. The calculations were based on carbohydrate structures containing the core unit with one fucose, two $\mathrm{N}$-acetylglucosamine and three mannose residues. Further additions were $0-4 \mathrm{~N}$-acetylneuraminic acid (sialic acid) residues (this number being represented by the first digit of the code) and $0-6$ $\mathrm{N}$-acetyl-lactosamine disaccharide units (this number being represented by the second digit of the code). The masses of the peptide moieties were calculated for amino acid sequences $\mathrm{Tyr}^{63}-$ Lys $^{97}$ (IIMGA and IIBGA), Tyr ${ }^{63}-\mathrm{Arg}^{98}$ (IIBGB) and $\mathrm{Ala}^{42}-\mathrm{Arg}^{62}$ of the carboxymethylated mutant lysozyme.

\begin{tabular}{|c|c|c|c|c|c|}
\hline \multirow{2}{*}{$\begin{array}{l}\text { Structure } \\
\text { (code) }\end{array}$} & \multicolumn{5}{|c|}{ Glycopeptides (\% of arbitary units) } \\
\hline & IIMGA & IIBGA & IIBGB & IVTMG & IVTBG \\
\hline 02 & 14.5 & & & 9.9 & 1.1 \\
\hline 03 & 7.6 & & & & \\
\hline 12 & 41.5 & 5.1 & 15.9 & 18.5 & 5.1 \\
\hline 13 & 12.9 & 5.4 & 7.1 & 7.7 & 4.4 \\
\hline 14 & 1.7 & & & 2.2 & 3.8 \\
\hline 15 & & & & & 4.8 \\
\hline 22 & 15.8 & 51.2 & 48.2 & 35.6 & 15.0 \\
\hline 23 & 3.6 & 21.4 & 17.9 & 11.9 & 8.1 \\
\hline 24 & & 5.2 & 3.7 & 3.1 & 8.8 \\
\hline 25 & & 2.8 & 1.1 & 1.2 & 8.3 \\
\hline 26 & & & & & 3.3 \\
\hline 33 & & 1.8 & & 1.4 & 4.7 \\
\hline 34 & & 1.9 & 1.3 & 1.7 & 12.7 \\
\hline 35 & & 4.3 & 3.2 & 1.3 & 12.1 \\
\hline 36 & & 1.0 & & & 3.8 \\
\hline 37 & & & & & 1.1 \\
\hline 45 & & & & & 1.5 \\
\hline 46 & & & & & 1.3 \\
\hline
\end{tabular}

The spectra obtained from glycopeptides of mono-glycosylated lysozyme (IIMGA and IVTMG) and from glycopeptides associated with site II in bis-glycosylated lysozyme (IIBGA and IIBGB) were much simpler than those of IVTBG. The predominant mass values in the former corresponded to mono- and bis-sialylated biantennary oligosaccharides. As an example, a mass spectrum of the glycopeptide IIBGB is shown in Figure 6. In Table 1 the relative abundance of oligosaccharides containing different numbers of sialic acid residues and $\mathrm{N}$-acetyl-lactosamine units was estimated from the relative frequency of arbitrary mass units in spectral measurements of the examined glycopeptides. The pattern of oligosaccharides in glycopeptide IIMGB (results not shown) was similar to those of IIBGA, IIBGB and IVTMG. A common feature of oligosaccharides associated with these glycopeptides, in distinction to IVTBG, was the predominance of smaller mass values corresponding to biantennary oligosaccharides with either no or few $N$-acetyl-lactosamine repeats (Table 1).

In the former group of glycopeptides, the mass of the main peak indicated the presence of bis-sialylated biantennary oligosaccharide (code 22). The amounts of larger oligosaccharides containing more $N$-acetyl-lactosamine units (codes 23-25) corresponded on average to approx. one third of the bis-sialylated biantennary oligosaccharide without repeats (code 22). Owing to the limits in quantitative interpretation of mass spectra it should be stressed that the relative contents of the elongated oligosaccharides that could be estimated from spectra of glycopeptides from mono-glycosylated lysozyme (Table 1 and Figure 6) agreed well with the intensity patterns of silver-stained bands of the lysozyme that the glycopeptides originated from (Figure 1) and of the radioactive bands of mono-glycosylated lysozyme contain- ing either glycosylation site that were inspected in this (Figure 7, below) and previous studies [9]. SDS/PAGE of monoglycosylated lysozyme containing more than two $\mathrm{N}$-acetyl-lactosamine units showed that it was nearly completely digested by endo- $\beta$ - $N$-acetylglucosaminidase $\mathrm{F} 2$, indicating that the larger species contained predominantly biantennary oligosaccharides with $\mathrm{N}$-acetyl-lactosamine repeats.

A significant portion of all glycopeptides contained incompletely sialylated oligosaccharides. The proportions of partially sialylated forms varied among the different glycopeptides. It was highest in IIMGA and lowest in IIBGA. The incomplete sialylation seemed to be related neither to the origin of the glycopeptides from mono- and bis-glycosylated lysozyme nor the separation in HPLC of site II glycopeptides into fractions A and B. Thus in one case A was less sialylated, while in the other case B was less sialylated. Papac et al. [17] have discussed in detail factors that contribute to loss of sialic acid from glycoconjugates during the preparation and analysis of samples in MALDI-TOF mass determinations. The present measurements were performed in the linear mode that produces spectra without desialylation. However, we could not exclude a loss of sialic acid residues due to interactions with the acidic matrix [17].

From comparisons of mass signal arbitary units in different samples it was apparent that in glycopeptides IVTBG the content of $\mathrm{N}$-acetyl-lactosamine repeats and of oligosaccharides with more than two antennae strongly exceeded those in the other glycopeptides (Table 1). These findings confirmed the interpretation of the shape of the HPLC peaks described above.

In glycopeptides IVTBG among the bis-sialylated species (structure codes 22-26 in Table 1) molecules with two and four $\mathrm{N}$-acetyl-lactosamine units were the most abundant. We assumed that the amount of the biantennary oligosaccharides with an increasing number of $\mathrm{N}$-acetyl-lactosamine units was monotonously decreasing just as in the case of the other glycopeptides shown in Table 1 . The relatively high apparent contents of oligosaccharides with 4-6 N-acetyl-lactosamine units (structure codes 24-26) in bis-sialylated glycopeptides IVTBG indicated that these were enriched in triantennary incompletely sialylated forms. Oligosaccharides with the codes 23-26 were likely to contain a decreasing proportion of bis-sialylated biantennary species with 1-4 additional $N$-acetyl-lactosamine repeats and an increasing proportion of bis-sialylated triantennary isomers. Quantitatively, the occurrence of the latter could be related to that of tris-sialylated triantennary oligosaccharides, among which those with one and two repeats (codes 34 and 36) were the major species (Figure 4). Similarly, oligosaccharides in peaks $12-15$ are likely to represent mono-sialylated biantennary forms that have been generated through in vitro desialylation [17] of glycopeptides $22-25$.

\section{Characterization of triantennary oligosaccharides in metabolically labelled Iysozyme}

MS analyses, shown above, indicated that in molecules that were glycosylated at both sites, tris-sialylated oligosaccharides comprising mostly the triantennary species were associated preferentially with the glycosylation site IV. We attempted to estimate the occurence of triantennary and elongated oligosaccharides using metabolic labelling, digestion of glycosylated lysozyme with endo- $\beta$ - $N$-acetylglucosaminidase $\mathrm{F} 2$ that cleaves biantennary, but not triantennary, complex oligosaccharides, and digestion with endo- $\beta$-galactosidase that removes antennae containing $N$-acetyl-lactosamine repeats. The interpretation was facilitated by quantitative PhosphorImager analysis (Figure 7). The quantification encompassed all visible bands, however, for 
the sake of brevity, values of only a few of them will be mentioned. In the control, $67 \%$ of the radioactivity was associated with mono-glycosylated lysozyme bearing complex oligosaccharide side chains (MGL in Figure 7). The most prominent band, lysozyme bearing a single bis-sialylated biantennary oligosaccharide without repeats, represented $40 \%$ of the total radioactivity. Its identity was based on resistance to endo- $\beta$-galactosidase (Figure 7) and endo- $N$-acetylglucosaminidase $\mathrm{H}$ [18], and sensitivity to endo- $\beta$ - $N$-acetylglucosaminidase F2 (Figure 7) and sialidase [9]. The bands migrating in SDS/PAGE in the monoglycosylated lysozyme area, behind the main band, represented $29 \%$ of the total radioactivity. This material was sensitive to endo- $\beta$-galactosidase and the cleavage products migrated in front of the main band that was resistant, as expected. In the digested sample, these bands, together, comprised $64 \%$ of the radioactivity, thus resembling the sum of radioactivity associated with the starting components of mono-glycosylated lysozyme. In the sample that had been digested with endo- $\beta$ - $N$-acetylglucosaminidase $\mathrm{F} 2$, little radioactivity was found to migrate immediately behind the main band where the resistant forms of mono-glycosylated lysozyme molecules with elongated triantennary oligosaccharides were expected, thus indicating scarcity of these derivatives in mono-glycosylated lysozyme.

PhosphorImager analysis of the group of bis-glycosylated lysozyme molecules (BGL) comprised $29 \%$ of the total radioactivity in the undigested sample. After removal of the repeats with endo- $\beta$-galactosidase these molecules migrated faster and, as expected, their quantity did not change. Due to poor band resolution, it was not possible to distinguish the products of digestion bearing different combinations of bi- and triantennary oligosaccharides with or without removed antennae. Digestion of metabolically labelled lysozyme II/IVT with endo- $\beta$ - $N$-acetylglucosaminidase F2 was expected to remove one or two biantennary oligosaccharides, thus forming lysozyme with one $\left(\mathrm{L}^{\prime}\right)$ or two $\left(\mathrm{L}^{\prime \prime}\right)$ fucosyl $N$-acetyglucosamine disaccharide units attached, respectively. As expected from the relative contents of mono- and bis-glycosylated lysozyme the amount of the former product exceeded that of the latter. A small portion of bisglycosylated lysozyme contained one cleavable biantennary oligosaccharide and one mannose-rich oligosaccharide, as indicated by the appearance of a product $\left(\mathrm{mL}^{\prime}\right)$ that migrated behind lysozyme with one mannose-rich oligosaccharide $(\mathrm{mL})$.

Portions of the bis-glycosylated molecules were completely or partially resistant to endo- $\beta-N$-acetylglucosaminidase F2. A minor fraction $(2 \%)$ appeared to contain two oligosaccharides even after the digestion (ttL). At least one of these was of complex type and contained $N$-acetyl-lactosamine repeats as indicated by the ladder-like array of the bands. It is possible that the resistance was due to the presence of one mannose-rich and one tri- or tetra-antennary oligosaccharide. The limited amount of this material precluded further analysis. A larger fraction of the resistant molecules ( $9 \%$ of the total radioactivity) migrated as a group of bands (tL in Figure 7) with apparent sizes intermediate between the typical mono- and bis-glycosylated lysozyme. This material was considered to originate from partially sensitive bis-glycosylated lysozyme, in which one site was sensitive and the other contained larger oligosaccharides resistant to endo- $\beta$ - $N$-acetylglucosaminidase $\mathrm{F} 2$. We assumed that the latter were the source of glycopeptide IVTBG that was the major species contaning triantennary and elongated oligosaccharides (Table 1). Similarly to mass analysis of glycopeptide IVTBG, the pattern of bands in the group tL in Figure 7 indicated that triantennary oligosaccharides at site IV in bis-glycosylated lysozyme comprise predominantly structures with one or more $N$-acetyl-lactosamine repeats. Thus the presence of elongated

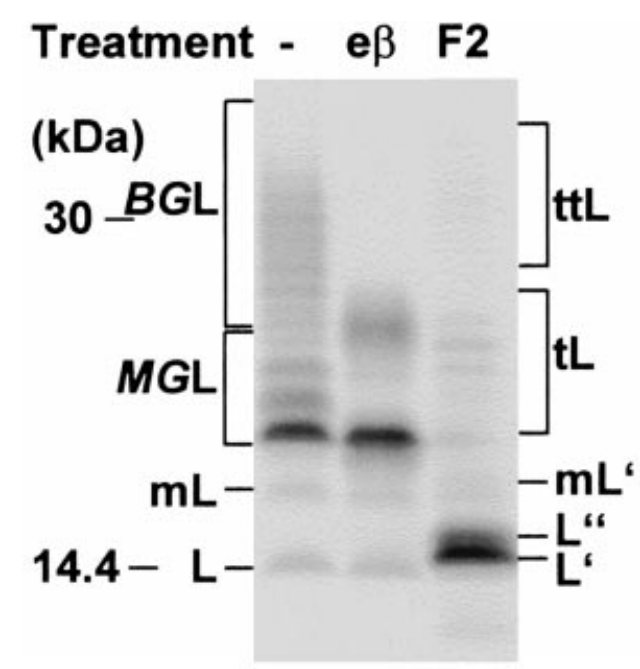

Figure 7 Digestion of biantennary oligosaccharides and of $\mathrm{N}$-acetyllactosamine repeats in bis-glycosylated lysozyme with glycosidases

Metabolically labelled II/IVT lysozyme was isolated by immunoprecipitation from secretions of transfected $\mathrm{CHO}$ cells. After solubilization, one aliquot was treated with endo- $\beta$ - $N$-acetylglucosaminidase $\mathrm{F} 2$, one with endo- $\beta$-galactosidase $(\mathrm{e} \beta)$ and one remained untreated $(-)$. The radioactivity associated with the different glycosylated forms of lysozyme was analysed by SDS/PAGE, fluorography and Phosphorlmaging. Positions of mono-(MGL) and bis-glycosylated lysozyme (BGL), lysozyme bearing mannose-rich $(\mathrm{mL})$, one (tL) or two (ttL) triantennary oligosaccharides, one $\left(\mathrm{L}^{\prime}\right)$ or two $\left(\mathrm{L}^{\prime}\right)$ fucosyl $\mathrm{N}$-acetylglucosamine disaccharides and one fucosyl $\mathrm{N}$-acetylglucosamine along with a mannose-rich oligosaccharide $\left(\mathrm{mL}^{\prime}\right)$ are indicated.

triantennary oligosaccharides in bis-glycosylated lysozyme II/ IVT was documented by results from both MALDI-TOF MS (Figure 4 and Table 1) and enzymic digestion (Figure 7). Furthermore, in the pool of bis-glycosylated lysozyme that was isolated by hydroxylapatite column chromatography, shown in Figure 1, the presence of triantennary oligosaccharides was confirmed by digesting this material with endo- $\beta$ - $N$-acetylglucosaminidase F2, separating the products by SDS/PAGE and visualizing them by silver staining (results not shown). In contrast, in mono-glycosylated lysozyme triantennary oligosaccharides were hardly detectable.

\section{DISCUSSION}

The biosynthesis of $\mathrm{N}$-acetyl-lactosamine repeats is a complex process and its regulation is poorly understood. Among factors that have been shown to enhance elongation of the repeats are the following: (1) synthesis of the $\beta$-1,6-linked third antenna in complex oligosaccharides $[6,7] ;(2)$ cell differentiation correlating with an increase in the activity of $\mathrm{N}$-acetylglucosaminyltransferase involved in elongation $[4,5]$; (3) slow transport through the Golgi apparatus in cells cultured at a decreased temperature [3]; (4) a lack of sialylation of complex oligosaccharides [9]. Furthermore, increased synthesis of $\mathrm{N}$-acetyllactosamine repeats is known to correlate with neoplastic transformation [6]. To our knowledge, the present data show for the first time that elongation of $N$-acetyl-lactosamine repeats at a particular carbohydrate attachment site is dependent on the presence of another oligosaccharide. In mutant lysozyme, glycosylation at $\mathrm{Asn}^{68}$ (site II) enhances the synthesis of $N$ acetyl-lactosamine repeats at $\mathrm{Asn}^{49}$ (site IV). This enhancement results at least in part from an increase in the synthesis of the third antenna that is the preferred site of elongation. The 
triantennary oligosaccharides in bis-glycosylated lysozyme contain mostly one or two repeats. Owing to the biological significance of the repeats as a scaffold in the synthesis of sialyl Lewis $^{\mathrm{x}}$ substances it should be of interest to determine the threedimensional structures of natural Lewis ${ }^{\mathrm{x}}$-bearing molecules and examine these for the presence of multiple carbohydrate residues that might be spaced similarly to glycosylation sites II and IV in the mutant lysozyme.

Carlsson and Fukuda [19] have reported that in lamp proteins $\mathrm{N}$-acetyl-lactosamine repeats are confined to particular glycosylation sites and suggested that their synthesis might depend on the accessibility of the sites, as well as on the threedimensional structure surrounding them. Our results with an engineered glycoprotein that is not related to lamp proteins extend the ideas of these authors and suggest that the synthesis of the repeats may be independent of any protein signal. As observed in bis-glycosylated lysozyme the processing signal seems to be associated with a carbohydrate rather than protein. Furthermore, the effect depends on the location of this carbohydrate. Thus as reported previously [11], in bis-glycosylated lysozyme the characteristic processing at site IV is observed if a second carbohydrate is located at site II $\left(\mathrm{Asn}^{68}\right)$, but not at site I $\left(\mathrm{Asn}^{22}\right)$.

Our findings are based on the separation of glycopeptides representing glycosylation sites II and IV from both mono- and bis-glycosylated lysozyme and their mass spectral analysis. We are aware that quantitative evaluation of arbitary units in mass spectra must be interpreted with caution. For this reason we should point out that in mono-glycosylated lysozyme, with predominantly biantennary oligosaccharides, the relative proportions of molecules with increasing numbers of $N$-acetyllactosamine repeats, as observed in either silver-stained gels (Figure 1) or PhosphorImaged metabolically labelled lysozyme (Figure 7) are similar to those found in MALDI-TOF spectra of corresponding glycopeptides (Table 1). It has been shown that during MALDI-TOF sample preparation and/or measurement a partial desialylation of oligosaccharides may occur [17]. We have observed previously that in $\mathrm{CHO}$ cells oligosaccharides in mono-glycosylated lysozyme appear to be nearly completely sialylated [9]. Therefore we assume that the contents of completely sialylated bi- and triantennary oligosaccharides at site IVT in bis-glycosylated lysozyme may be higher than can be anticipated from the present mass spectrum results. Although mass spectral analysis indicates how many $N$ acetyl-lactosamine units are present it does not differentiate between isomers. However, from mass values the minimum number of sialic acids and, thus of antennae, can be estimated. Our finding on the increase in branching of the oligosaccharides at site IV in lysozyme II/IVT is based on the increase in both the contents of tris-sialylated oligosacharides and the resistance of oligosaccharides towards endo- $\beta$ - $N$-acetylglucosaminidase F2.

Two or more $\beta-1,3-N$-acetylglucosaminyltransferases are likely to participate in the synthesis of the elongating units in $N$-linked oligosaccharides. The known candidates are the elongating $\beta-1,3-N$-acetylglucosaminyltransferase [20] and a novel $\beta-1,3-N$ acetylglucosaminyltransferase that is related to $\beta$-1,3-galactosyltransferases [21]. In complex oligosaccharides elongation proceeds preferentially at the $\beta$-1,6-linked antenna [22] and the synthesis of this antenna in glycoproteins is increased in metastatic tumour cells [23]. This so-called Warren-Glick phenomenon has been explained by a cancer-related increase in the activity of $N$-acetylglucosaminyltransferase V [24,25]. Our results suggest that this enzyme may preferentially modify particular carbohydrate side chains in the context of an additional glycosylation. In lysozyme II/IVT the modification of the oligosaccharide at the glycosylation site IV appears to result from the presence of carbohydrate at glycosylation site II. Thus a question may be raised whether isolated $\beta-1,6-N$-acetylglucosaminyltransferase $\mathrm{V}$ can be activated by oligosaccharides that are attached to the substrate protein near the acceptor carbohydrate. This enzyme has been the subject of numerous enzymological studies [26-31]. However, to our knowledge its activation by carbohydrate side chains has yet to be examined.

This work was supported by the Deutsche Forschungsgemeinschaft, SFB 286, the Alexander von Humboldt-Stiftung (to A. H.) and the Fonds der Chemischen Industrie. We thank Gertraud Jarosch for excellent technical help, Evelin Heinemann for secretarial assistance and Barbara Schedding for amino acid sequence analyses.

\section{REFERENCES}

1 Ley, K. (1996) Molecular mechanisms of leukocyte recruitment in the inflammatory process. Cardiovasc. Res. 32, 733-742

2 McEver, R. P. (1997) Selectin-carbohydrate interactions during inflammation and metastasis. Glycoconjugate J. 14, 585-591

3 Wang, W.-C., Lee, N., Aoki, D., Fukuda, M. N. and Fukuda, M. (1991) The poly-Nacetyl-lactosamines attached to lysosomal membrane glycoproteins are increased by the prolonged association with the Golgi complex. J. Biol. Chem. 266, 23185-23190

4 Lee, N., Wang, W.-C. and Fukuda, M. (1990) Granulocytic differentiation of HL-60 cells is associated with increase of poly- $\mathrm{N}$-acetyl-lactosamine in Asn-linked oligosaccharides attached to human lysosomal membrane glycoproteins. J. Biol. Chem. 265, 20476-20487

5 Radons, J., Faber, V., Buhrmester, H., Völker, W., Horejsi, V. and Hasilik, A. (1992) Stimulation of the biosynthesis of lactosamine repeats in glycoproteins in differentiating U937 cells and its suppression in the presence of $\mathrm{NH}_{4} \mathrm{Cl}$. Eur. J. Cell Biol. 57, 184-192

6 Yousefi, S., Higgins, E., Daoling, Z., Pollex-Kruger, A., Hindsgaul, 0. and Dennis, J. W. (1991) Increased UDP-GIcNAc:Gal beta-1,3-GalNAc-R (GIcNAc to GaINAc) beta$1,6-\mathrm{N}$-acetylglucosaminyltransferase activity in metastatic murine tumor cell lines. Control of polylactosamine synthesis. J. Biol. Chem. 166, 1772-1782

7 Koenderman, H. L., Koppen, P. L. and van den Eijnden, D. H. (1987). Biosynthesis of polylactosaminoglycans. Novikoff ascites tumor cells contain two UDP-GlcNAc: betagalactoside beta-1-6-N-acetylglucosaminyltransferase activities. Eur. J. Biochem. 166, 199-208

8 Capon, C., Laboisse, C. L., Wieruszenski, J.-N., Maoret, J. J., Augeron, C. and Fournet, B. (1992) Oligosaccharide structures of mucins secreted by the human colonic cancer cell line CL.16E. J. Biol. Chem. 267, 19248-19257

9 Hummel, M., Hedrich, H. C. and Hasilik, A. (1997) Elongation of $\mathrm{N}$-acetyl-lactosamine repeats in biantennary oligosaccharides. Eur. J. Biochem. 245, 428-433

10 Fernandes, B., Sagman, U., Auger, M., Demetrio, M. and Dennis, I. W. (1991) Beta1,6-branched oligosaccharides as a marker of tumor progression in human breast and colon neoplasia. Cancer Res. 51, 718-723

11 Melcher, R., Grosch, H.-W., Grosse, O. and Hasilik, A. (1998) Increased elongation of $\mathrm{N}$-acetyl-lactosamine repeats in doubly glycosylated lysozyme with a particular spacing of the glycosylation sites. Glycoconjugate J. 15, 987-993

12 Gupta, D. K., Theisen, N., von Figura, K. and Hasilik, A. (1985) Comparison of biosynthesis and transport of lysozyme and cathepsin D in U937 monocytes. Biochim. Biophys. Acta, 847, 217-222

13 Laemmli, U. K. (1970) Cleavage of structural proteins during the assembly of the head of bacteriophage T4. Nature (London) 227, 680-685

14 Heukeshoven, J. and Dernick, R. (1988) Improved silver staining procedure for fast staining in PhastSystem Development Unit. I. Staining of sodium dodecyl sulfate gels. Electrophoresis 9, 28-32

15 Beavis, R. C. and Chait, B. T. (1996) Matrix-assisted laser desorption ionization massspectrometry of proteins. Methods Enzymol. 270, 519-551

16 Tsarbopoulos A., Bahr U., Pramanik B. N. and Karas M. (1997) Glycoprotein analysis by delayed extraction and post-source decay MALDI TOF MS. Int. J. Mass Spectrom. Ion Processes 169/170, 251-261

17 Papac, D. I., Wong, A. and Jones, A. J. S. (1996) Analysis of acidic oligosaccharides and glycopeptides by matrix-assisted laser desorption/ionization time-of-flight mass spectrometry. Anal. Chem. 68, 3215-3223

18 Horst, M., Harth, N. and Hasilik, A. (1990) Biosynthesis of glycosylated human lysozyme mutants. J. Biol. Chem. 266, 13914-13919

19 Carlsson, S. R. and Fukuda, M. (1990) The polylactosaminoglycans of human lysosomal membrane glycoproteins lamp-1 and lamp-2. Localization on the peptide backbones. J. Biol. Chem. 265, 20488-20495 
20 Sasaki, K., Kurata-Miura, K., Ujita, M., Angata, K., Nakagawa, S., Sekine, S., Nishi, T. and Fukuda, M. (1997) Expression cloning of cDNA encoding a human $\beta-1,3-N$ acetylglucosaminyltransferase that is essential for poly- $\mathrm{N}$-acetyl-lactosamine synthesis. Proc. Natl. Acad. Sci. U.S.A. 94, 14294-14299

21 Zhou, D., Dinter, A., Gallego, R. G., Kamerling, J. P., Vliegenthart, J. F. G., Berger, E. G. and Hennet, T. (1999) A $\beta$-1,3- $N$-acetylglucosaminyltransferase with poly- $N$ acetyl-lactosamine synthase activity is structurally related to $\beta-1,3$ galactosyltransferases. Proc. Natl. Acad. Sci. U.S.A. 96, 406-411

22 van den Eijnden, D. H., Koenderman, A. H. and Schiphorst, W. E. (1988) Biosynthesis of blood group i-active polylactosaminoglycans. Partial purification and properties of an UDP-GIcNAc: $N$-acetyl-lactosaminide beta-1,3- $N$-acetylglucosaminyltransferase from Novikoff tumor cell ascites fluid. J. Biol. Chem. 263, 12461-12471

23 Dennis, J. W., Laferte, S., Waghorne, C., Breitman, M. L. and Kerbel, R. S. (1987) Beta-1,6 branching of Asn-linked oligosaccharides is directly associated with metastasis. Science 236, 582-585

24 Kobata, A. (1989) Altered glycosylation of surface glycoproteins in tumor cells and its clinical application. Pigm. Cell Res. 2, 304-308

25 Dennis, J. W., Granovsky, M. and Warren, C. E. (1999) Glycoprotein glycosylation and cancer progression. Biochim. Biophys. Acta 1473, 21-34

26 Kanie, O., Crawley, S. C., Palcic, M. M. and Hindsgaul, 0. (1993) Acceptor-substrate recognition by $\mathrm{N}$-acetylglucosaminyltransferase-V: critical role of the $4^{\prime \prime}$-hydroxyl group in beta-D-GlcpNAc-(1 $\rightarrow$ 2)-alpha-D-Manp(1 $\rightarrow$ 6)-beta-D- Glcp-0R. Carbohydr. Res. 243, 139-164

27 Khan, S. H., Crawley, S. C., Kanie, O. and Hindsgaul, 0. (1993) A trisaccharide acceptor analog for $\mathrm{N}$-acetylglucosaminyltransferase $\mathrm{V}$ which binds to the enzyme but sterically precludes the transfer reaction. J. Biol. Chem. 268, 2468-2473

28 Gu, J., Nishikawa, A., Tsuruoka, N., Ohno, M., Yamaguchi, N., Kangawa, K. and Taniguchi, N. (1993) Purification and characterization of UDP-N-acetylglucosamine: alpha-6-D-mannoside beta-1,6 $\mathrm{N}$-acetylglucosaminyl- transferase $(\mathrm{N}$ acetylglucosaminyltransferase $V$ ) from a human lung cancer cell line. J. Biochem. (Tokyo) 113, 614-619

29 Do, K. Y., Fregien, N., Pierce, M. and Cummings, R. D. (1994) Modification of glycoproteins by $\mathrm{N}$-acetylglucosaminyltransferase $\mathrm{V}$ is greatly influenced by accessibility of the enzyme to oligosaccharide acceptors. J. Biol. Chem. $\mathbf{2 6 9}$ 23456-23464

30 Brockhausen, I., Reck, F., Kuhns, W., Khan, S., Matta, K. L., Meinjohanns, E., Paulsen, H., Shah, R. N., Baker, M. A. and Schachter, H. (1995) Substrate specificity and inhibition of UDP-GIcNAc:GlcNAc beta 1,2 Man alpha 1,6R beta 1,6- $N$ acetylglucosaminyltransferase $\mathrm{V}$ using synthetic substrate analogues. Glycoconjugate J. 12, 371-379

31 Zhang, N., Peng, K. C., Chen, L., Puett, D. and Pierce, M. (1997) Circular dichroic spectroscopy of $\mathrm{N}$-acetylglucosaminyltransferase $\mathrm{V}$ and its substrate interactions. J. Biol. Chem. 272, 4225-4229

Received 17 September 1999/6 March 2000; accepted 7 April 2000 